\title{
Auditory Verbal Working Memory as a Predictor of Speech Perception in Modulated Maskers in Listeners With Normal Hearing
}

DOI:

10.1044/2017_JSLHR-S-16-0105

\section{Document Version}

Accepted author manuscript

Link to publication record in Manchester Research Explorer

Citation for published version (APA):

Millman, R. E., \& Mattys, S. L. (2017). Auditory Verbal Working Memory as a Predictor of Speech Perception in Modulated Maskers in Listeners With Normal Hearing. Journal of Speech, Language, and Hearing Research, 60(5), 1236-1245. https://doi.org/10.1044/2017_JSLHR-S-16-0105

\section{Published in:}

Journal of Speech, Language, and Hearing Research

\section{Citing this paper}

Please note that where the full-text provided on Manchester Research Explorer is the Author Accepted Manuscript or Proof version this may differ from the final Published version. If citing, it is advised that you check and use the publisher's definitive version.

\section{General rights}

Copyright and moral rights for the publications made accessible in the Research Explorer are retained by the authors and/or other copyright owners and it is a condition of accessing publications that users recognise and abide by the legal requirements associated with these rights.

\section{Takedown policy}

If you believe that this document breaches copyright please refer to the University of Manchester's Takedown Procedures [http://man.ac.uk/04Y6Bo] or contact uml.scholarlycommunications@manchester.ac.uk providing relevant details, so we can investigate your claim.

\section{OPEN ACCESS}




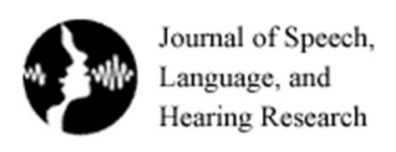

\section{Auditory verbal working memory as a predictor of speech perception in modulated maskers in normal-hearing listeners}

\begin{tabular}{|r|l|}
\hline Journal: & Journal of Speech, Language, and Hearing Research \\
\hline Manuscript ID & JSLHR-S-16-0105.R2 \\
\hline Manuscript Type: & Research Article \\
\hline Date Submitted by the Author: & 11 -Oct-2016 \\
\hline Complete List of Authors: & $\begin{array}{l}\text { Millman, Rebecca; University of Manchester, Manchester Centre for } \\
\text { Audiology and Deafness } \\
\text { Mattys, Sven; University of York, Department of Psychology }\end{array}$ \\
\hline Keywords: & Memory, Speech perception, Aging \\
\hline \multicolumn{2}{|c}{} \\
\hline
\end{tabular}




\section{Auditory verbal working memory as a predictor of speech perception in modulated maskers in normal-hearing listeners ${ }^{\text {a) }}$}

Rebecca E. Millman ${ }^{\text {b) }}$ and Sven L. Mattys ${ }^{\text {c) }}$

a) Portions of this work were presented at the Third International Conference on Cognitive Hearing Science for Communication, Linköping, Sweden, 2015.
${ }^{b)}$ Manchester Centre for Audiology and Deafness, School of Health Sciences, Faculty of Biology, Medicine, and Health, University of Manchester, Manchester, M13 9PL, UK.

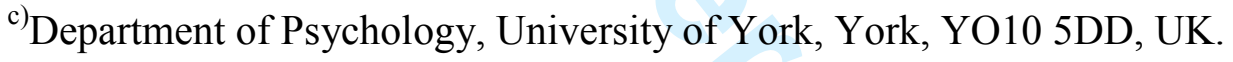

Submitted to: Journal of Speech, Language, and Hearing Research

Running title: Auditory memory for speech in noise

Author to whom correspondence should be addressed: Rebecca E. Millman

Telephone: +44 (0) $1612753387 \quad$ Fax: $+44(0) 1612753373$

Electronic-mail: rebecca.millman@manchester.ac.uk 


\begin{abstract}
Purpose: Background noise can interfere with our ability to understand speech. Working memory capacity (WMC) has been shown to contribute to the perception of speech in modulated noise maskers. WMC has been assessed with a variety of auditory and visual tests, often pertaining to different components of working memory. This study assessed the relationship between speech perception in modulated maskers and components of auditory verbal working memory (AVWM) over a range of signal-to-noise ratios.
\end{abstract}

Method: Speech perception in noise and AVWM were measured in 30 listeners (age range 31-67 years) with normal hearing. AVWM was estimated using forward digit recall, backward digit recall, and non-word repetition.

\begin{abstract}
Results: After controlling for the effects of age and average pure-tone hearing threshold, speech perception in modulated maskers was related to individual differences in the phonological component of working memory (as assessed by non-word repetition) but only in the least favorable SNR. The executive component of working memory (as assessed by backward digit) was not predictive of speech perception in any conditions.
\end{abstract}

\footnotetext{
Conclusions: AVWM is predictive of the ability to benefit from temporal dips in modulated maskers: Listeners with greater phonological WMC are better able to correctly identify sentences in modulated noise backgrounds.
} 


\section{Introduction}

Older adult listeners frequently experience difficulties in understanding speech presented in a noisy background. Difficulties may result from reduced audibility (e.g., Humes, 2002), age (e.g., Dubno, Horwitz, \& Ahlstrom, 2002), perceptual deficits in temporal resolution, spectral resolution, or cochlear compression (e.g., Gregan, Nelson, \& Oxenham, 2013), and deficits in the cognitive systems underpinning speech perception (CHABA, 1988; Wingfield, Tun, \& McCoy, 2005). Among the cognitive abilities related to the perception of speech in noisy backgrounds, working memory has attracted substantive attention (e.g., Gatehouse, Naylor, \& Eberling, 2003; Lunner, 2003; Akeroyd, 2008; Rönnberg, Rudner, Lunner, \& Zekveld, 2010; Mattys, Davis, \& Bradlow, 2012; Füllgrabe \& Rosen, 2016). Working memory may be conceptualized as the system that underpins the temporary storage and manipulation of information required for cognitive tasks (e.g., Baddeley, 1992).

One view argues that age-related differences in cognition can be accounted for by deficits in auditory processing (e.g., Schneider \& Pichora-Fuller, 2000; McCoy, Tun, Cox, Colangelo, Stewart, \& Wingfield, 2005; Humes, Busey, Craig, \& Kewley-Port, 2015). On this account, age-related decline in auditory processing depletes working memory resources so that fewer resources are available for storage and manipulation of information. Consistent with this view, age-related differences in cognitive tasks may be largely eliminated when the perceptual difficulty of the tasks are taken into account (e.g., Pichora-Fuller, Schneider, \& Daneman, 1995; Murphy, Craik, Li, \& Schneider, 2000).

An alternative view, as proposed by the Ease of Language Understanding (ELU) model (e.g., Rönnberg, 2003; Rönnberg et al., 2010; Rönnberg, Danielsson, Rudner, 
Arlinger, Sternäng, Wahlin, \& Nilsson, 2011; Rönnberg, Lunner, Zekveld, Sörqvist, Danielsson, Lyxell, Dahlström, Signoret, Stenfelt, Pichora-Fuller, \& Rudner, 2013), argues that working memory is always deployed when speech is presented against a noisy background, even at favorable signal-to-noise ratios (SNRs). The ELU model predicts reliance on working memory in perceptually adverse conditions because working memory is thought to: 1) Compensate for the ambiguity caused by degraded phonological representations, 2) Contribute to predictive coding, either before or during the early portions of a spoken stimulus, and 3) Facilitate explicit semantic expectations of a sentence as it unfolds (e.g., Rönnberg et al., 2013). The role of working memory may become even more important when speech is presented in a fluctuating masker because listeners with greater cognitive capacity are more able to take advantage of the temporal dips in the masker (e.g., Gatehouse et al., 2003). Working memory may play a greater role in fluctuating maskers in older listeners because age-related deficits in speech perception are exacerbated when the background noise is modulated (e.g., Takahasi \& Bacon, 1992; Dubno et al., 2002). The dependence on working memory is increased further when the masker consists of speech rather than meaningless fluctuating noise (Koelewijn, Zekveld, Festen, Rönnberg, \& Kramer, 2012; Zekveld, Rudner, Johnsrude, \& Rönnberg, 2013a). In such challenging listening situations, working memory is thought to support maintenance of a mental representation of a spoken sentence, whilst knowledge of language and context are recruited to fill the gaps in the degraded auditory signal.

The exact contribution of working memory to speech perception under adverse conditions remains controversial, however. Part of the debate centers on handling confounds such as age (e.g., Wingfield, Stine, Lahar, \& Aberdeen, 1988; Salthouse, 
1994), pure-tone average thresholds (PTAs), and stimulus audibility (Baldwin \& Ash, 2011; McCoy et al., 2005; Wingfield et al., 2005). Other issues are related to the validity of the tests used for measuring working memory. For instance, although the reading-span test (R-SPAN, Daneman \& Carpenter, 1980; Baddeley, Logie, Nimmo-Smith, \& Brereton, 1985) is a widely used measure of (visual) verbal working memory in hearing research (e.g., Pichora-Fuller et al., 1995; Lunner, 2003; Akeroyd, 2008; Füllgrabe, Moore, \& Stone, 2015; Smith \& Pichora-Fuller, 2015), recent work (e.g., Sörqvist, Ljungberg, \& Ljung, 2010; Sörqvist \& Rönnberg, 2012) has questioned the use of RSPAN as the sole suitable measure of working memory.

Because both reading and listening span tasks are highly correlated with reading comprehension in young normal-hearing adults under quiet listening conditions (e.g., Daneman \& Carpenter, 1980), it is often assumed that verbal working memory for language comprehension is modality-independent. However, evidence of modalityspecific working memory (Humes, Burk, Couchlin, Busey, \& Strauser, 2007) has motivated comparisons of the R-SPAN with its auditory equivalent, the listening span (LSPAN; e.g., Koelewijn et al., 2012; Zekveld, Festen, \& Kramer, 2013b; Besser, Koelewijn, Zekveld, Kramer, \& Festen, 2013). Specifically, data have shown age-related deficits in performance on the L-SPAN but not on the R-SPAN (e.g. Pichora-Fuller et al., 2005; Baldwin \& Ash, 2011). Moreover, L-SPAN performance is not related to speech perception in noise in older listeners with normal hearing (NH listeners), whereas RSPAN performance is (Koelewijn et al., 2012). Besser et al. (2013) also noted that LSPAN scores are generally higher than R-SPAN scores in NH listeners (Koelewijn et al., 2012; Zekveld et al., 2013b). These discrepancies between R-SPAN and L-SPAN 
outcomes further question the reliance on SPAN tests in assessing the contribution of working memory to speech perception in noise (e.g., Sörquist \& Ronnberg, 2012).

The primary objective of the present study was to determine whether age-related changes in AVWM are associated with deficits in understanding speech in noise in listeners with normal hearing. A speech in noise task was chosen as the dependent measure in order to provide a listening situation that would promote reliance on working memory, i.e., the ability to take advantage of the amplitude minima in modulated maskers to detect speech cues. When temporal fluctuations are imposed on an unmodulated noise masker, listeners sometimes benefit from the temporal dips in the masker, i.e., they exhibit speech masking release (MR). Listeners may need components of AVWM to retain glimpses of speech revealed during the temporal dips. Additional cognitive factors, including linguistic abilities (e.g., Benard, Mensink, \& Başkent, 2014; Avivi-Reich, Daneman, \& Schneider, 2014), which also involve memory processes, may also be used to make inferences about the target speech based on partial acoustic and linguistic cues obtained during the temporal dips.

Given the complex nature of the R-SPAN and L-SPAN tests, which tax both the central executive (for processing) and phonological loop (for storage) components of working memory (e.g., Baddeley et al., 1985; Gathercole, Pickering, Ambridge, \& Wearing, 2004), any relationship between verbal working memory capacity measured using these tests and the ability to understand speech in noise remains ill-defined.

In this study, we aimed to isolate the sub-component(s) of AVWM that contribute(s) to the ability to benefit from speech MR and speech perception in modulated noise. Accordingly, standardized tests of AVWM, namely, forward digit 
recall, backward digit recall, and non-word repetition, were used. These tests may be considered in terms of a gradient of processing demands (e.g., Daneman \& Merikle, 1996), with forward digit recall requiring little processing demand, backward digit span requiring more processing demand, and non-word repetition requiring the greatest processing demand. However, an alternative view based on Baddeley's (1992) model of working memory proposes that these tests probe different sub-processes in AVWM: Forward digit recall and backward digit recall both tap into the phonological component of working memory, whereas backward digit recall additionally relies on an attentiondemanding manipulation of the digit sequence by the executive control (e.g., Alloway, Gathercole, Willis, \& Adams, 2004; Gathercole et al., 2004). In that conceptualization, backward digit recall loads onto the same factor as more complex working memory such as the SPAN tests (e.g., Alloway et al., 2004; Gathercole et al., 2004). The phonological component of working memory is a common underlying factor in both digit recall and non-word repetition, but these two tasks are also supported by long-term lexical knowledge (e.g. Gathercole, 1995). Long-term lexical knowledge may play a lesser role in non-word repetition than digit recall, depending on the degree of "wordlikeness" of the non-words (e.g., Gathercole, Willis, Emslie, \& Baddeley, 1991; Gathercole, 1995). The aim of using these different tests of AVWM was to clarify the role of sub-processes in AVWM in speech perception in modulated maskers.

\section{Methods}

\section{Listeners}


Thirty listeners, all native English speakers, participated in the study. Listeners (11 male) ranged in age from 31 to 67 years (mean age $=53.5$ years, s.d. $=9.4$ years). All listeners provided written informed consent and were paid for their participation in the study.

Pure-tone air-conduction audiometric thresholds were measured in accordance with the procedures recommended by the British Society of Audiology (British Society of Audiology, 2004). The listeners had audiometrically normal hearing in both ears, defined as audiometric thresholds of no more than $20 \mathrm{~dB}$ HL for octave frequencies between 0.5 and $4 \mathrm{kHz}$. Individual and mean audiograms of NH listeners are shown in Fig 1. Audiometric thresholds were measured for frequencies of $0.5,1,2$, and $4 \mathrm{kHz}$ only, because the stimuli were bandpass-filtered with cut-off frequencies of 0.5 and $4 \mathrm{kHz}$ using a $6^{\text {th }}$-order Butterworth filter and a linear-phase FIR digital filter (Vickers, Moore, \& Baer, 2001).

\section{Insert Figure 1 about here}

\section{Procedure}

Audiometric thresholds, AVWM capacity, and speech perception were measured in one session. Listeners were seated in a double-walled sound-attenuating booth. For the measures of AVWM and speech perception, stimuli were delivered diotically through Sennheiser HD 650 headphones. Stimuli were played through an E-MU soundcard using custom MATLAB (The MathWorks Inc., Natick, MA) routines. The overall stimulus level was $65 \mathrm{~dB}$ SPL. Depending on the task, responses were made either orally (AVWM) or typed manually by the participants using a keyboard (speech perception). 


\section{Speech masking release (MR)}

The speech stimuli were IEEE sentences (Rothauser, Chapman, Guttman, Silbiger, Hecker, Urbanek, Nordby, \& Weinstick, 1969) spoken by five female speakers. Some aspects of the experimental design for the measurement of speech perception were driven by considerations for an accompanying neuroimaging experiment, which is not included in the present study. The entire list of IEEE sentences was sorted based on duration: One hundred and twenty sentences of the IEEE shortest sentences (mean duration $=2.22 \mathrm{~s}, \mathrm{~s} . \mathrm{d} .=0.06 \mathrm{~s}$ ) were used for the behavioral testing and a further thirty sentences were used for training prior to behavioral testing. Sentences contained four or five key words (mean number of key words $=4.92$, s.d. $=0.27$ ).

Sentences were normalised for long-term root mean square (RMS) amplitude. Key word identification was measured in a masking noise that was spectrally shaped to match the long-term power spectrum of the sentences. The noise was either unmodulated or $100 \%$ modulated with a $2-\mathrm{Hz}$ square-wave (50 \% duty cycle). The "on" and "off" periods of the 2-Hz square-wave masker were both $250 \mathrm{~ms}$. The on/off slopes of the square-wave were shaped with 5-ms cosine-squared ramps. The noise was shaped after scaling and the level of the modulated noise during the "on" periods was the same as that of the unmodulated noise (Gregan et al., 2013). The modulation frequency (4 Hz) of the modulated noise was similar to the speech rate ( $4 \mathrm{~Hz}$, e.g., Hughes, 2013$)$ of the IEEE sentences.

Speech MR was defined as the difference in the correct response rate between the unmodulated and modulated masker conditions. In the case of speech MR, the optimal 
masker modulation rate is dependent on the speech material. A modulation frequency of 8-10 Hz usually provides the greatest amount of speech MR (e.g., Miller \& Licklider, 1950; Gustafsson \& Arlinger, 1994). We chose $2 \mathrm{~Hz}$ because results from previous studies show that a $2-\mathrm{Hz}$ square-wave masker provides substantial benefit from sentencelevel speech MR (e.g., Nelson, Jin, Carney, \& Nelson, 2003). Different noise samples were selected for each presentation. When the noise was modulated, the first period of the noise was always "on" and therefore the first 250-ms portion of each sentence was masked. The speech-in-noise stimuli were ramped on and off using a 25-ms raised cosine function.

One method for measuring speech perception in noise is to compare the signal-tonoise ratio (SNR) required for $50 \%$ correct performance in the unmodulated masker with the SNR required for $50 \%$ correct performance in the modulated masker, i.e., the speech reception threshold $\left(\mathrm{SRT}_{50}\right)$. In the case of speech $\mathrm{MR}, \mathrm{SRT}_{50}$ is often difficult to calculate accurately because listeners with NH usually perform well even at low SNRs when the masker is modulated: For some listeners, performance in modulated noise may not fall below $50 \%$ correct, even for very unfavorable SNRs (e.g., Gregan et al., 2013). Therefore, an accepted alternative method (e.g., Nelson et al., 2003; Gregan et al., 2013; Füllgrabe et al., 2015) is to calculate the difference in performance between the unmodulated and modulated masker at fixed levels of SNR, which is the method we used here. SNRs were fixed at $-4,-8$, and $-12 \mathrm{~dB}$. Negative SNRs were chosen because they result in more speech MR (e.g., Bernstein \& Grant, 2009; Bernstein \& Brungart, 2011; Gregan et al., 2013). Twenty sentences were randomly assigned to each of three SNR conditions $(-4,-8$, and $-12 \mathrm{~dB})$ and two masker conditions (unmodulated, modulated). 
The order of the SNR and masker conditions was randomized prior to the start of each participant's testing. Participants were instructed to type the words they could understand using a computer keyboard.

Speech perception was measured as the percentage of words correctly transcribed. Individual scores for correct identification of keywords were transformed into rationalized arcsine units (RAU) (Studebaker, 1985) for statistical analyses. Unless specified, the results depicted in figures and in the text are the untransformed percentages of keywords correctly identified.

\section{Auditory Verbal Working Memory (AVWM)}

The primary aim of the present study was to isolate the components of AVWM that contribute to speech MR and speech perception in modulated noise in NH listeners. To this end, auditory stimuli from a subset of the Automated Working Memory Assessment (AWMA) tests (Pearson Education Limited, London, United Kingdom, 2007) were employed. The AWMA tests used were non-word repetition (NWR), forward digit recall (Digit), and backward digit recall (BackDigit). As mentioned previously, these AWMA tests may be considered in terms of a gradient of processing demands (e.g., Daneman \& Merikle, 1996), with Digit entailing minimal processing demands and NWR requiring the greatest processing demands. They can also be described as probing different sub-components of AVWM. NWR is assumed to assess the phonological component of working memory (e.g., Gathercole et al., 1991; Gathercole et al., 2004). Both Digit and BackDigit assess the phonological component of working memory as well, but, importantly, BackDigit is also thought to require executive control (e.g., Alloway et al., 2004; Gathercole et al., 2004) because the digits must undergo active 
processing in order to be recalled in reverse order. The order of the AWMA tests was counterbalanced across participants.

For each test, number sequences or non-word sequences of increasing length were presented to listeners. The starting number of items was two. There were six trials per length. The test was discontinued after 3 incorrect responses for a given length. For the Digit and BackDigit tests, responses were scored as incorrect if verbal responses did not correspond exactly to the target sequence of numbers. For the NWR test, responses were scored as incorrect if the verbal response differed phonemically from the target non-word by one phoneme or more. The maximum possible score was 36 for the NWR test, 48 for the Digit test, and 36 for the BackDigit test.

\section{Results}

\section{Speech perception in noise maskers}

Mean keyword identification scores are shown in Fig. 2. Consistent with previous work (e.g., Nelson et al., 2003; Gregan et al., 2013), speech perception in the presence of an unmodulated masker at low SNRs was poor. The lower SNRs (-8 and $-12 \mathrm{~dB})$ resulted in floor performance in the unmodulated masker condition. Given the floor performance in those conditions, speech MR was only measured for $-4 \mathrm{~dB}$ SNR. For that condition, a paired $t$-test (2-tailed) confirmed that listeners benefitted from speech masking release, that is, speech perception in the modulated masker condition was significantly better than in the unmodulated masker condition [35.6\%, s.d. $=9.3 \%, t(29)=20.34, p<0.005]$.

The results also showed that speech perception in the modulated masker condition improved as the SNR increased: From $23.8 \%$ (s.d. $=6.5 \%$ ) at $-12 \mathrm{~dB}$ SNR to $46.3 \%$ 
$($ s.d. $=9.2 \%)$ at $-4 \mathrm{~dB}$ SNR. An Analysis of Variance (ANOVA) confirmed the increase in performance as a function of $\operatorname{SNR}[F(2,58)=124.11, p<0.005]$.

\section{Insert Fig. 2 about here}

The MR effect at $-4 \mathrm{~dB}$ SNR is consistent with previous studies that used unmodulated and square-wave modulated noise to measure speech MR (e.g., Nelson et al., 2003; Gregan et al., 2013). Notable methodological differences in the present study compared to those of Gregan et al. (2013) include: 1) A lower masker modulation frequency for the modulated masker (2-Hz in our study vs. $10-\mathrm{Hz}$ in theirs) and 2) The temporal relationship of the speech and the masker (speech and masker were gated on and off together in our study vs. the speech signal was temporally centered within a longer duration masker in theirs). A 2-Hz square-wave modulated masker results in less speech MR than a 10-Hz square-wave modulated masker (e.g., Nelson et al., 2003). However, in the present study, the modulated noise was always in phase with the sentence onset, which would make the amplitude minima in the modulated noise predictable, given that only one modulation rate was tested. The predictability of the temporal dips in the modulated masker would improve absolute performance in the modulated masker condition and increase the amount of speech MR, relative to a modulated masker with a random starting phase.

\section{Link between AVWM, hearing thresholds, and age}


Fig. 3 shows the mean scores on the AVWM tests of NWR, Digit, and BackDigit.

\author{
Insert Fig. 3 about here
}

Hierarchical multiple regression analyses were employed to further explore the predictive value of Age and PTA on each one of the sub-components of AVWM considered separately. Age was entered first, followed by PTA. Table I shows that Age and PTA showed different patterns of association with the sub-components of AVWM. NWR scores were reliably predicted by Age $\left(R^{2}=0.25, p=0.005\right)$, but PTA did not further increase the model fit $\left(\Delta R^{2}=0.05, p=0.16\right)$. Forward digit scores were also reliably predicted by Age in the first step of the model $\left(R^{2}=0.15, p=0.03\right)$, but adding PTA did not improve the fit $\left(\Delta R^{2}=0, p=0.99\right)$. As for BackDigit, Age did not make a contribution to performance $\left(R^{2}=0.05, p=0.25\right)$ in the first step of the model. However, in the second step of the model, PTA did add to the model $\left(\Delta R^{2}=0.14, p=0.04\right)$.

In sum, consistent with the finding that working memory capacity declines with age (e.g., Wingfield et al., 1988; Salthouse, 1994), the present results show that the subcomponents of AVWM that relate to the phonological component of working memory (forward digit recall, non-word repetition) were affected by age. There was no significant effect of age on backward digit recall, which is in contrast with some previous studies (e.g., Tun, McCoy, \& Wingfield, 2009). It is possible that the association between age and auditory forward and backward digit recall identified in those studies was confounded with age-related increases in hearing thresholds. In the present study, all 
listeners had clinically-defined normal hearing (i.e., $\leq 20 \mathrm{~dB} \mathrm{HL}$ ), and there was no significant correlation between age and PTA ( $r=0.024, p=0.90$, 2-tailed significance).

We also found that the executive component of working memory, as measured by backward digit recall, was influenced by hearing thresholds. This was in sharp contrast with the lack of effect of hearing thresholds on forward digit recall and non-word repetition, which are measures of the phonological component of AVWM that requires little executive control (e.g., Gathercole et al., 2004). These differential predictive values of age and PTA support the idea that the AVWM tests used here probed different subprocesses in AVWM (e.g., Alloway et al., 2004; Gathercole et al., 2004), rather than representing a gradient of processing demands (Daneman \& Merikle, 1996). We return to this distinction in the next section.

\section{Link between AVWM and speech perception in modulated maskers}

Hierarchical multiple regression models were used to assess AVWM predictors of speech MR and speech perception in modulated maskers. Variables thought to affect speech perception in noise, namely Age (Dubno et al., 2002; George, Festen, \& Houtgast, 2006) and PTA (e.g., Bacon, Opie, \& Montoya, 1998; Desloge, Reed, Braida, Perez, \& Delhorne, 2010), were entered in the first step of the model. The AVWM tests were included in a stepwise-fashion in a second step because the relative contributions of subcomponents of AVWM to speech perception in modulated maskers are unknown.

Analyses were carried out for MR at $-4 \mathrm{~dB}$ SNR only because 1) This SNR is comparable with that used by Gregan et al. (2013, -5 dB SNR) to assess perceptual predictors of speech MR, and 2) The results for the $-4 \mathrm{~dB}$ SNR condition were not 
affected by floor performance. Results are shown in Table 2 (speech MR, -4 dB SNR). The first step, which included Age $(p=0.50)$ and PTA $(p=0.10)$, did not significantly predict speech MR $\left(R^{2}=0.12, p=0.19\right)$. The AVWM scores did not add to the model fit (NWR, $p=0.46$; Digit, $p=0.33$; BackDigit, $p=0.64$ ).

Regression analyses were also carried out for speech perception in the modulated noise masker at each SNR $(-4,-8$, and $-12 \mathrm{~dB})$. Age and PTA were included in the first step of the model and the scores on the AVWM tests (NWR, Digit, and BackDigit) were added in the second step. These analyses test a central prediction of the ELU model (e.g., Rönnberg, 2013), namely that working memory supports speech perception in noise, even at more favorable SNRs. At an SNR of $-4 \mathrm{~dB}$ (Table 3), the first step of the model, including Age $(p=0.04)$ and PTA $(p=0.17)$, was predictive of performance $\left(R^{2}=0.20\right.$, $p=0.046$ ). The AVWM scores did not increase the model fit (NWR, $p=0.13$; Digit, $p=$ 0.4; BackDigit, $p=0.82$ ). At $-8 \mathrm{~dB}$ SNR (Table 4$)$, neither Age $(p=0.29)$, nor PTA ( $p=$ $0.43)$ predicted performance $\left(R^{2}=0.06, p=0.41\right)$. The AVWM scores did not improve the model fit (NWR, $p=0.1$; Digit, $p=0.47$; BackDigit, $p=0.36$ ). At $-12 \mathrm{~dB}$ SNR (Table 5), Age $(p=0.12)$ and PTA $(p=0.03)$ were predictive of performance $\left(R^{2}=0.23\right.$, $p=0.03$ ). In the second step, only the phonological component of working memory, as measured by NWR $(p=0.048)$, added to the model fit $\left(\Delta R^{2}=0.11, p=0.048\right)$ for an SNR of $-12 \mathrm{~dB}$ (Digit, $p=0.33$, BackDigit, $p=0.48$ ). Fig. 4 shows that better speech perception in a modulated masker at $-12 \mathrm{~dB}$ SNR was associated with higher NWR scores $(p=0.04)$. 
In sum, these analyses show that the phonological component of working memory (NWR) is the sub-component of AVWM that contributes most to speech perception in modulated noise. The lack of association between BackDigit and speech-in-noise perception suggests that individual differences in central executive processing are not significantly related to speech perception in modulated noise maskers.

\section{General Discussion}

Speech perception in noise is influenced by both perceptual factors (e.g. PichoraFuller et al., 1995; Gregan et al., 2013) and cognitive factors (CHABA, 1988; Wingfield, et al., 2005; Rönnberg et al., 2013). There is some evidence of a link between (visual) verbal working memory capacity and the ability of both normal-hearing (Koelewijn, et al., 2012; Zekveld et al., 2013a; Füllgrabe \& Rosen, 2016) and hearing-impaired listeners (Lunner, 2003; Foo, Rudner, Rönnberg, \& Lunner, 2007; Rönnberg et al., 2010, 2011, 2013; Rudner, Rönnberg, and Lunner, 2011) to understand speech in noisy backgrounds. To date, however, there has been limited evidence that auditory verbal working memory (AVWM) contributes to understanding speech in noise (for reviews, see Akeroyd, 2008; Besser et al., 2013). In the present study, we examined the relationship between individual sub-components of AVWM and the ability of NH listeners to take advantage of the temporal dips in modulated maskers to aid speech perception. We hypothesized that individual differences in AVWM would be related to listeners' ability to understand speech played in modulated maskers. Indeed, understanding sentences played against modulated background noise requires successive elements of a sentence, especially those 
revealed during the amplitude minima in the masker, to be identified and remembered until the syntactic structure of the sentence can be parsed and its meaning inferred (Lyxell \& Rönnberg, 1989; Rönnberg et al., 2013).

Within the Ease of Language Understanding framework (ELU, Rönnberg, 2003; Rönnberg et al., 2011, 2013), phonological input is matched to stored phonological representations in semantic long-term memory. Because ambiguity between input and stored representations is higher under perceptually adverse conditions, working memory is recruited to resolve the mismatch. The ELU model predicts that working memory contributes to speech perception in noise even at favorable SNRs (e.g., Rönnberg et al., 2013). Therefore, we assessed the relationship between speech perception in modulated maskers and components of AVWM over a range of signal-to-noise ratios. We found that individual differences in the phonological component of working memory were linked with the ability to perceive speech in modulated noise only when the SNR was very unfavorable (i.e., $-12 \mathrm{~dB}$ ). Thus, our results support the link between working memory and speech perception put forward in the ELU model, but they do not support the claim that this relationship is manifest at more favorable SNRs (i.e., -4 or $-8 \mathrm{~dB}$ ), at least in listeners with normal hearing and with the present stimuli.

Individual differences on the non-word repetition (NWR) test of AVWM were associated with speech perception in modulated noise. NWR is a measure of the phonological component of working memory and, to some extent, long-term lexical knowledge (Gathercole \& Adams, 1994; Gathercole, 1995). Listeners may rely on the interplay between the phonological component of working memory and lexical knowledge to support the perception of speech in modulated noise. In order for listeners 
to benefit from temporal dips in modulated maskers, the phonological component of working memory may be needed to retain glimpses of locally and temporarily meaningless information in working memory. Together with a contribution from longterm lexical knowledge, the phonological component of working memory may contribute to improving speech perception in modulated noise through retention of speech cues acquired during the temporal dips.

This view of an interplay between the phonological component of working memory and long-term lexical knowledge to support speech perception in modulated noise is consistent with ELU predictions (e.g., Rönnberg et al., 2011, 2013). Indeed, the network supporting speech perception in noise relies heavily on working memory and is also critically dependent on additional cognitive resources, including linguistic abilities (Benard et al., 2014), lexical and linguistic compensation (e.g., Avivi-Reich et al., 2015), and phonological and lexical representations from semantic long-term memory (Rönnberg et al., 2011; 2013). For example, linguistic context, retrieved from long-term memory, rather than working memory, contributes to the restoration of interrupted speech (Benard et al., 2014; Nagaraj \& Knapp, 2015). Long-term semantic memory resources may be deployed to interact with the information that has already been decoded in the phonological component of working memory, i.e., the glimpses acquired during temporal dips in modulated masker, to facilitate improved speech perception in modulated noise.

The results presented here suggest that uncovering a relationship between speech perception in noise and AVWM depends on selecting the appropriate test of AVWM. In this study, only the phonological component of working memory, as measured using nonword repetition, was associated with speech perception in modulated noise. Considered in 
terms of a gradient of processing demands (e.g., Daneman \& Merikle, 1996), NWR may be associated with speech perception in -12 dB SNR because NWR may entail the greatest processing demands of the three AVWM tests we used. However, the variation in the predictive values of Age and PTA on the individual AVWM tests suggests that these tests might instead probe sub-components of AVWM with different requirements for executive control, rather than simply different gradients of processing demands.

We did not find an association between speech perception in modulated noise and individual differences in the central executive component of working memory, as measured by backward digit recall. If individual differences in central executive functions are not predictive of speech perception in modulated noise, this may explain why some previous studies have failed to identify a relationship between complex (visual) verbal working memory measures involving the central executive, i.e., the reading span test, and the perception of interrupted speech (e.g., Nagaraj \& Knapp, 2015; Shafiro, Sheft, Risley, \& Gygi, 2015).

\section{Summary}

The present study provides support for differential roles of sub-components of auditory verbal working memory in the abilities of listeners with normal hearing to use the speech cues available during the temporal dips in modulated background noise. Specifically, individual differences in the phonological component of working memory, rather than the central executive component, were associated with speech perception in modulated noise under adverse listening conditions. 


\section{ACKNOWLEDGMENTS}

This study was funded in part by the Wellcome Trust [ref: 105624] through the Centre for Chronic Diseases and Disorders (C2D2) at the University of York, UK. 


\title{
REFERENCES
}

Akeroyd, M. A. (2008). Are individual differences in speech perception related to individual differences in cognitive ability? A survey of twenty experimental studies with normal and hearing impaired adults. International Journal of Audiology, 47, 53-71.

\begin{abstract}
Alloway, T. P., Gathercole, S. E., Willis, C., \& Adams, A-M. (2004). A structural analysis of working memory and related cognitive skills in young children. Journal of Experimental Child Psychology, 87, 85-406.
\end{abstract}

Avivi-Reich, M., Daneman, M, \& Schneider, B. A. (2014). How age and linguistic competence alter the interplay of perceptual and cognitive factors when listening to conversations in a noisy environment. Frontiers in Systems Neuroscience, 8, 21.

Bacon, S. P., Opie, J. M., \& Montoya, D. Y. (1998). The effects of hearing loss and noise masking on the masking release for speech in temporally complex backgrounds. Journal of Speech Language, and Hearing Research, 41, 549-563.

\footnotetext{
Baddeley, A., Logie, R., Nimmo-Smith, I., \& Brereton, N. (1985). Components of fluent reading. Journal of Memory \& Language, 24, 119-131.
}

Baddeley, A. (1992). Working memory. Science, 255, 556-559. 


\begin{abstract}
Baldwin, C. L., \& Ash, I. K. (2011). Impact of sensory acuity on auditory working memory span in young and older adults. Psychology of Aging, 26, 85-91.
\end{abstract}

\author{
Benard, M., Mensink, J., \& Başkent, D. (2014). Individual differences in top-down \\ restoration of interrupted speech: Links to linguistic and cognitive abilities. \\ Journal of the Acoustical Society of America, 135, EL88-EL94.
}
Bernstein, J. G. W., \& Grant, K. W. (2009). Auditory and auditory-visual intelligibility of speech in fluctuating maskers for normal-hearing and hearing- impaired listeners. Journal of the Acoustical Society of America, 125, 3358-3372.
Bernstein, J. G. W., \& Brungart, D. S. (2011). Effects of spectral smearing and temporal fine-structure distortion on the fluctuating-masker benefit for speech at a fixed signal-to-noise ratio. Journal of the Acoustical Society of America, 130, 473-488.
Besser, J., Koelewijn, T., Zekveld, A. A., Kramer, S. E., \& Festen, J. M. (2013). How linguistic closure and verbal working memory relate to speech recognition in noise - A review. Trends in Amplification, 17, 75-93.

British Society of Audiology (2004). Recommended procedure: Pure tone air and bone 
conduction thresholds audiometry with and without masking and determination of uncomfortable loudness levels. Reading, UK: British Society of Audiology.

CHABA (Committee on Hearing, Bioacoustics, and Biomechanics). (1988). Speech understanding and aging. Journal of the Acoustical Society of America, 83, 859895.

Daneman, M., \& Carpenter, P. A. (1980). Individual differences in working memory and reading. Journal of Verbal Learning and Verbal Behavior, 19, 450-466.

Daneman, M., \& Merikle, P. M. (1996). Working memory and language comprehension: A meta-analysis. Psychnomic Bulletin \& Review, 3, 422-433.

\author{
Desloge, J. G., Reed, C. M., Braida, L. D., Perez, Z. D., \& Delhorne, L. A. (2010). \\ Speech reception by listeners with real and simulated hearing impairment: effects \\ of continuous and interrupted noise. Journal of the Acoustical Society of America, \\ $128,342-359$.
}
Dubno, J. R., Horwitz, A. R., \& Ahlstrom, J. B. (2002). Benefit of modulated maskers for speech recognition by younger and older adults with normal hearing. Journal of the Acoustical Society of America, 111, 2897-2907.

Foo, C., Rudner, M., Rönnberg, J., \& Lunner, T. (2007). Recognition of speech in 
noise with new hearing instrument compression release settings requires explicit cognitive storage and processing capacity. Journal of the American Academy of Audiology, 18, 553-566.

Füllgrabe, C., Moore, B. C. J., \& Stone, M. A. (2015). Age-group differences in speech identification despite matched audiometrically normal hearing: contributions from auditory temporal processing and cognition. Frontiers in Aging Neuroscience, 6, 347.

Füllgrabe, C., \& Rosen, S. (2016). On the (un)importance of working memory in speech-in-noise processing for listeners with normal hearing thresholds. Frontiers in Psychology, 7, 1268.

Gatehouse, S., Naylor, G., \& Eberling, C. (2003). Benefits from hearing aids in relation to the interaction between the user and the environment. International Journal of Audiology, 42, S77-85.

Gathercole, S. E., Willis, C., Emslie, H., \& Baddeley, A. D. (1991). The influences of number of syllables and wordlikeness on children's repetition of nonwords. Applied Psycholinguistics, 12, 349-367.

Gathercole, S. E., \& Adams, A.-M. (1994). Children's phonological working memory: 
Contributions of long-term knowledge and rehearsal. Journal of Memory \& Language, 33, 672-388.

Gathercole, S. E. (1995). Is nonword repetition a test of phonological memory or lexical knowledge? It all depends on the nonwords. Memory \& Cognition, 23, 83-94.

Gathercole, S. E., Pickering, S. J., Ambridge, B., \& Wearing, H. (2004). The structure of working memory from 4 to 15 years of age. Developmental Psychology. 40, 177-190.

George, E. L. J., Festen, J. M., \& Houtgast, T. (2006). Factors affecting masking release for speech in modulated noise for normal-hearing and hearing impaired listeners. Journal of the Acoustical Society of America, 120, 2295-2311.

\footnotetext{
Gregan, M. J., Nelson, P. B., \& Oxenham, A. J. (2013). Behavioral measures of cochlear compression and temporal resolution as predictors of speech masking release in hearing-impaired listeners. Journal of the Acoustical Society of America, 134, 2895-2912.
}

Gustafsson, H. A., \& Arlinger, S. D. (1994). Masking of speech by amplitude-
modulated noise. Journal of the Acoustical Society of America, 95, 518-529.

Hughes, M. L. (2013). Objective measures in cochlear implants, $1^{\text {st }}$ ed. (Plural 
Publishing, San Diego).

Humes, L. E. (2002). Factors underlying the speech-recognition performance of elderly hearing-aid wearers. Journal of the Acoustical Society of America, 112, 11121132.

Humes, L. E., Burk, M. H., Coughlin, M. P., Busey, T. A., \& Strauser, L. E. (2007). Auditory speech recognition and visual text recognition in younger and older adults: similarities and differences between modalities and the effects of presentation rate. Journal of Speech, Language, and Hearing Research, 50, 283303.

Humes, L. E., Busey, T. A., Craig, J., \& Kewley-Port, D. (2015). Are age-related changes in cognitive function driven by age-related changes in sensory processing? Attention Perception and Psychophysics, 75, 508-524.

Koelewijn, T., Zekveld, A. A., Festen, J. M., Rönnberg, J., \& Kramer, S. E. (2012). Processing load induced by informational masking is related to linguistic abilities. International Journal of Otolaryngology, 2012, 1-11.

Lunner, T. (2003). Cognitive function in relation to hearing aid use. International Journal of Audiology, 42, S49-58. 
Lyxell, B., \& Rönnberg, J. (1989). Information-processing skills and speechreading. British Journal of Audiology, 23, 339-347.

Mattys, S. L., Davis, M. H., \& Bradlow, A. R. (2012). Speech recognition in adverse conditions: A review. Language and Cognitive Processes, 27, 953-978.

\title{
McCoy, S. L., Tun, P. A., Cox, C. L., Colangelo, M., Stewart, R. A., \& Wingfield, A. (2005). Hearing loss and perceptual effort: Downstream effects on older adults’ memory for speech. The Quarterly Journal of Experimental Psychology, 58, 22- 33.
}

Miller, G. A., \& Licklider, J. C. R. (1950). The intelligibility of interrupted speech. Journal of the Acoustical Society of America, 22, 167-173.

\author{
Murphy, D. R., Craik, F. I. M., Li, K. \& Schneider, B. A. (2000). Comparing the \\ effects of aging and background noise on short-term memory performance. \\ Psychology and Aging, 14, 44-59.
}
Nagaraj, N. K., \& Knapp, A. N. (2015). No evidence of relation between working memory and perception of interrupted speech in young adults. Journal of the Acoustical Society of America, 138, EL145-EL150.

Nelson, P. B., Jin, S. H., Carney, A. E., \& Nelson, D. A. (2003). Understanding speech 
in modulated interference: Cochlear implant users and normal-hearing listeners. Journal of the Acoustical Society of America, 113, 961-968.

Pichora-Fuller, M. K., Schneider, B. A., \& Daneman, M. (1995). How young and old adults listen to and remember speech in noise. Journal of the Acoustical Society of America, 97, 593-608.

Rönnberg, J. (2003). Cognition in the hearing impaired and deaf as a bridge between signal and dialogue: a framework and a model. International Journal of Audiology, 42, S68-S76.

Rönnberg, J., Rudner, M., Lunner, T., \& Zekveld, A. A. (2010). When cognition kicks in: Working memory and speech understanding in noise. Noise \& Health, $12,263$.

\section{Rönnberg, J., Danielsson, H., Rudner, M., Arlinger, S., Sternäng, O., Wahlin, Å., \&} Nilsson, L-G. (2011). Hearing loss is negatively related to episodic and semantic long-term memory but not to short-term memory. Journal of Speech, Language, and Hearing Research, 54, 705-726.

Rönnberg, J., Lunner, T., Zekveld, A. A, Sörqvist, P., Danielsson, H., Lyxell, B., 
Dahlström, Ö, Signoret, C., Stenfelt, S., Pichora-Fuller, M. K., \& Rudner, M. (2013). The Ease of Language Understanding (ELU) model: theoretical, empirical, and clinical advances. Frontiers in Systems Neuroscience, 7, 31.

\author{
Rothauser, E., Chapman, W., Guttman, N., Silbiger, H., Hecker, M., Urbanek, G., \\ Nordby, K., \& Weinstock, M. (1969). IEEE recommended practice for speech \\ quality measurements. IEEE Transactions in Acoustics, 17, 225-246.
}
Rudner, M., Rönnberg, J., \& Lunner, T. (2011). Working memory supports listening in noise for persons with hearing impairment. Journal of the American Academy of Audiology, 22, 156-167.

Salthouse, T. A. (1994). The aging of working memory. Neuropsychology, 8, 535-543.
Schneider, B. A., \& Pichora-Fuller, M. K. (2000). Implications of perceptual deterioration for cognitive aging research," The Handbook of Aging and Cognition, $2^{\text {nd }}$ ed. (Erlbaum, New York).
Shafiro, V., Sheft, S., Risley, R., \& Gygi, B. (2015). Effects of age and hearing loss on the intelligibility of interrupted speech. Journal of the Acoustical Society of America, 137, 745-756.

Sörqvist, P., Ljungberg, J. K., \& Ljung, R. (2010). A sub-process view of working 
memory capacity: Evidence from effects of speech on prose memory. Memory, $18,310-326$.

Sörqvist, P., \& Rönnberg, J. (2012). Episodic long-term memory of spoken discourse masked by speech: What is the role for working memory capacity? Journal of Speech, Language, and Hearing Research, 55, 210-218.

Smith, S. L., \& Pichora-Fuller, M. K. (2015). Associations between speech understanding and auditory and visual tests of verbal working memory: Effects of linguistic complexity, task, age, and hearing loss. Frontiers in Psychology, 6, 1394.

Studebaker, G. A. (1985). A “rationalized" arcsine transform. Journal of Speech and Hearing Research, 28, 455-462.

Takahashi, G. A., \& Bacon, S. P. (1992). Modulation detection, modulation masking, and speech understanding in noise in the elderly. Journal of Speech and Hearing Research, 35, 1410-1421.

Tun, P. A., McCoy, S., \& Wingfield. A. (2009). Aging, hearing acuity, and the attentional costs of effortful listening. Psychology of Aging, 24, 761-766.

Vickers, D. A., Moore, B. C. J., \& Baer, T. (2001). Effects of low-pass filtering on the 
intelligibility of speech in quiet for people with and without dead regions at high frequencies. Journal of the Acoustical Society of America, 110, 1164-1175.

\author{
Wingfield, A., Stine, E. L., Lahar, C. J., \& Aberdeen, J. S. (1988). Does the capacity \\ of working memory change with age? Experimental Aging Research, 14, 103- \\ 107. \\ Wingfield, A., Tun, P. A., \& McCoy, S. L. (2005). Hearing loss in older adulthood: \\ what it is and how it interacts with cognitive performance. Current Directions in \\ Psychological Science, 14, 144-148.
}

\author{
Zekveld, A. A., Rudner, M., Johnsrude, I. S., Ronnberg, J. (2013a). The effects of \\ working memory capacity and semantic cues on the intelligibility of speech in \\ noise. Journal of the Acoustical Society of America, 134, 2225-2234.
}

Zekveld, A. A., Festen, J. M., \& Kramer, S. E. (2013b). Task difficulty differentially affects two measures of processing load: The pupil response during sentence processing and delayed cued recall of the sentences. Journal of Speech, Language, and Hearing Research, 56, 1156-1165. 


\title{
FIGURE CAPTIONS
}

\author{
FIG. 1. Pure-tone air-conduction audiometry for the left ear (left panels) and right ear \\ (right panels) of the listeners. The solid grey lines represent individual audiometric \\ thresholds. The solid black lines represent mean audiometric thresholds for the listeners \\ and error bars represent one standard error of the mean (SEM). The dashed dark grey line \\ indicates the threshold of $20 \mathrm{~dB}$ HL that was considered the limit of normal hearing.
}

\begin{abstract}
FIG. 2. Mean accuracy (\%) for keyword identification of IEEE sentences as a function of SNR. Sentences were presented in an unmodulated noise masker (grey line) and a squarewave-gated noise masker (black line). Error bars show +/- one SEM.
\end{abstract}
FIG. 3. Mean AWMA raw scores. Memory tests were non-word repetition (NWR), forward digit recall (Digit) and backward digit recall (BackDigit). Error bars show +/- one SEM.

FIG. 4. Partial regression plots of the relationship between non-word repetition (NWR) and speech perception in modulated noise at -12 dB SNR (Speech Identification (RAU), $12 \mathrm{~dB}$ SNR), after controlling for the effects of Age and PTA. 


\section{TABLE I.}

Linear model of predictors (Age and PTA) of sub-components of AVWM (NWR, Digit, and BackDigit). Model parameters shown include standardized beta coefficients $(\beta)$ and the significance value $(p)$. The $R^{2}$ for the initial model step along with the change in $R^{2}\left(\Delta R^{2}\right)$ for the second step of the model are also reported. Values in bold indicate results at $p \leq 0.05$.

\begin{tabular}{|c|c|c|c|c|c|c|c|}
\hline \multirow[b]{2}{*}{ Model } & & \multicolumn{2}{|c|}{ NWR } & \multicolumn{2}{|c|}{ Digit } & \multicolumn{2}{|c|}{ Backdigit } \\
\hline & & $\beta$ & $p$ & $\beta$ & $p$ & $\beta$ & $p$ \\
\hline \multirow[t]{3}{*}{ Step 1} & Age & -0.5 & 0.005 & -0.39 & 0.03 & -0.22 & 0.25 \\
\hline & & \multicolumn{2}{|c|}{$R^{2}=0.25, p=0.005$} & \multicolumn{2}{|c|}{$R^{2}=0.15, p=0.03$} & \multicolumn{2}{|c|}{$R^{2}=0.05, p=0.25$} \\
\hline & & $\beta$ & $p$ & $\beta$ & $p$ & $\beta$ & $p$ \\
\hline \multirow[t]{3}{*}{ Step 2} & Age & -0.5 & 0.004 & -0.39 & 0.03 & -0.21 & 0.24 \\
\hline & PTA & -0.23 & 0.16 & 0.001 & 0.99 & -0.37 & 0.04 \\
\hline & & \multicolumn{2}{|c|}{$\Delta R^{2}=0.05, p=0.16$} & \multicolumn{2}{|c|}{$\Delta R^{2}=0, p=0.99$} & \multicolumn{2}{|c|}{$\Delta R^{2}=0.14, p=0.04$} \\
\hline
\end{tabular}




\section{TABLE 2.}

Linear model of predictors of speech MR for an SNR of $-4 \mathrm{~dB}$ for NH listeners. See Table 1 legend for details. Values in bold indicate results at $p \leq 0.05$.

\begin{tabular}{llcc}
\hline \hline \multicolumn{3}{c}{ speech MR (-4 dB SNR) } \\
Model & & $\beta$ & $p$ \\
\hline Step 1 & Age & -0.12 & 0.50 \\
& PTA & -0.31 & 0.10 \\
& & \\
$R^{2}=0.12$ for Step 1 $(p=0.19)$. \\
Excluded & NWR & -0.16 & 0.46 \\
& Digit & 0.2 & 0.33 \\
& BackDigit & -0.1 & 0.64 \\
& & & \\
\hline \hline
\end{tabular}

30

31

32

33

34

35

36

37

38

39

40

41

42

43

44

45

46

47

48

49

50

51

52

53

54

55

56

57

58

59

60 


\section{TABLE 3.}

Linear model of predictors of speech perception in a modulated masker for an SNR of $-4 \mathrm{~dB}$.

See Table 1 legend for details. Values in bold indicate results at $p \leq 0.05$.

\begin{tabular}{|c|c|c|c|}
\hline \multicolumn{4}{|c|}{$\begin{array}{l}\text { speech perception in modulated masker } \\
\qquad(-4 \mathrm{~dB} \text { SNR })\end{array}$} \\
\hline Model & & $\beta$ & $p$ \\
\hline \multirow{2}{*}{ Step 1} & Age & -0.38 & 0.04 \\
\hline & PTA & -0.24 & 0.17 \\
\hline \multirow[t]{3}{*}{ Excluded } & NWR & 0.32 & 0.13 \\
\hline & Digit & 0.16 & 0.40 \\
\hline & BackDigit & 0.05 & 0.82 \\
\hline \multicolumn{4}{|c|}{$R^{2}=0.20$ for Step $1(p=0.046)$} \\
\hline
\end{tabular}


2

3

4

TABLE 4.

Linear model of predictors of speech perception in a modulated masker for an SNR of $-8 \mathrm{~dB}$.

See Table 1 legend for details. Values in bold indicate results at $p \leq 0.05$.

\begin{tabular}{llcc}
\hline \hline \multicolumn{4}{c}{ speech perception in modulated masker } \\
\multicolumn{4}{c}{$(-8 \mathrm{~dB}$ SNR $)$} \\
Model & & $\beta$ & $p$ \\
\hline Step 1 & Age & -0.2 & 0.29 \\
& PTA & -0.15 & 0.43 \\
& & & \\
Excluded & NWR & 0.37 & 0.10 \\
& Digit & 0.15 & 0.47 \\
& BackDigit & 0.19 & 0.36 \\
$R^{2}=0.06$ for Step 1 $(p=0.41)$. \\
\end{tabular}

30

31

32

33

34

35

36

37

38

39

40

41

42

43

44

45

46

47

48

49

50

51

52

53

54

55

56

57

58

59

60 


\section{TABLE 5.}

Linear model of predictors of speech perception in a modulated masker for an SNR of -12

dB. See Table 1 legend for details. Values in bold indicate results at $p \leq 0.05$.

\begin{tabular}{|c|c|c|c|}
\hline \multicolumn{4}{|c|}{$\begin{array}{l}\text { speech perception in modulated masker } \\
(-12 \mathrm{~dB} \text { SNR })\end{array}$} \\
\hline Model & & $\beta$ & $p$ \\
\hline \multirow{2}{*}{ Step 1} & Age & -0.27 & 0.12 \\
\hline & PTA & -0.39 & $\mathbf{0 . 0 3}$ \\
\hline \multirow[t]{3}{*}{ Excluded } & NWR & 0.4 & 0.048 \\
\hline & Digit & 0.06 & 0.75 \\
\hline & BackDigit & -0.14 & 0.94 \\
\hline \multicolumn{4}{|c|}{$R^{2}=0.23$ for Step $1(p=0.03)$} \\
\hline \multirow[t]{3}{*}{ Step 2} & Age & -0.07 & 0.71 \\
\hline & PTA & -0.3 & 0.08 \\
\hline & NWR & 0.4 & 0.048 \\
\hline \multirow[t]{2}{*}{ Excluded } & Digit & -0.21 & 0.33 \\
\hline & BackDigit & -0.13 & 0.48 \\
\hline \multicolumn{4}{|c|}{$\Delta R^{2}=0.11$ for Step $1(p=0.048)$} \\
\hline
\end{tabular}




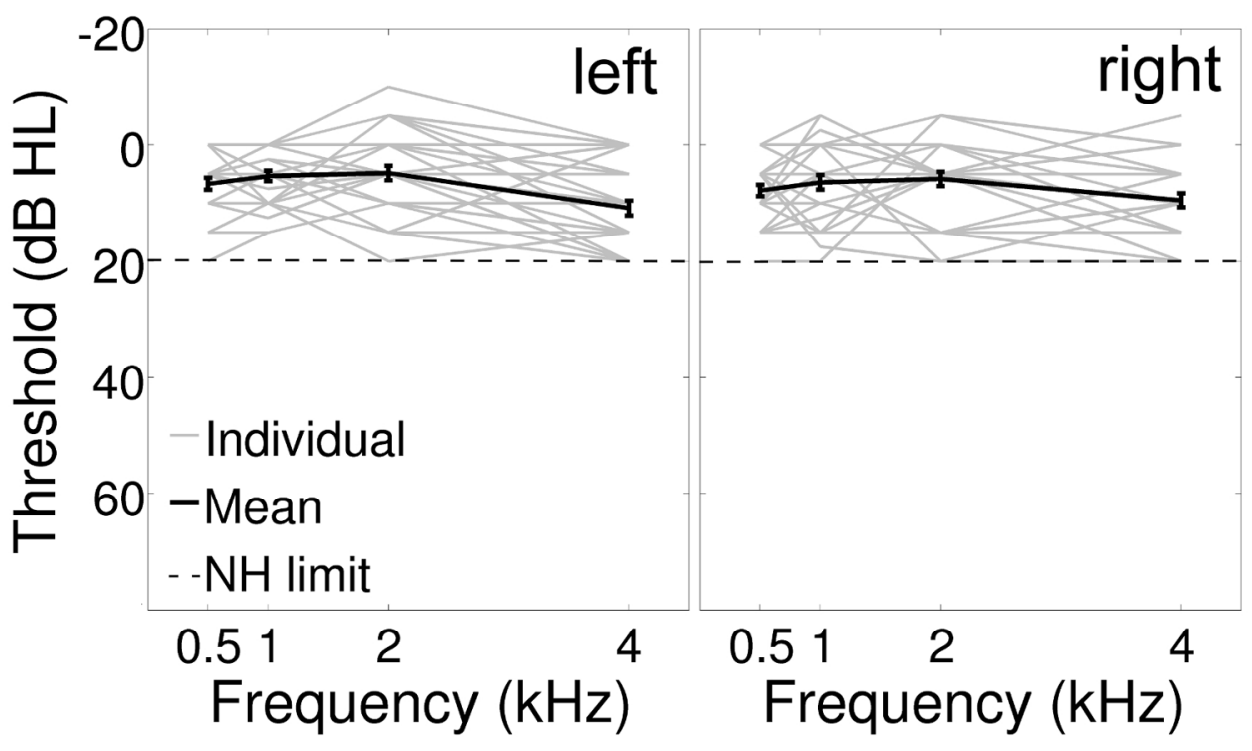

FIG. 1. Pure-tone air-conduction audiometry for the left ear (left panels) and right ear (right panels) of the listeners. The solid grey lines represent individual audiometric thresholds. The solid black lines represent mean audiometric thresholds for the listeners and error bars represent one standard error of the mean (SEM). The dashed dark grey line indicates the threshold of $20 \mathrm{~dB} H \mathrm{HL}$ that was considered the limit of normal hearing.

$152 \times 92 \mathrm{~mm}(300 \times 300 \mathrm{DPI})$ 


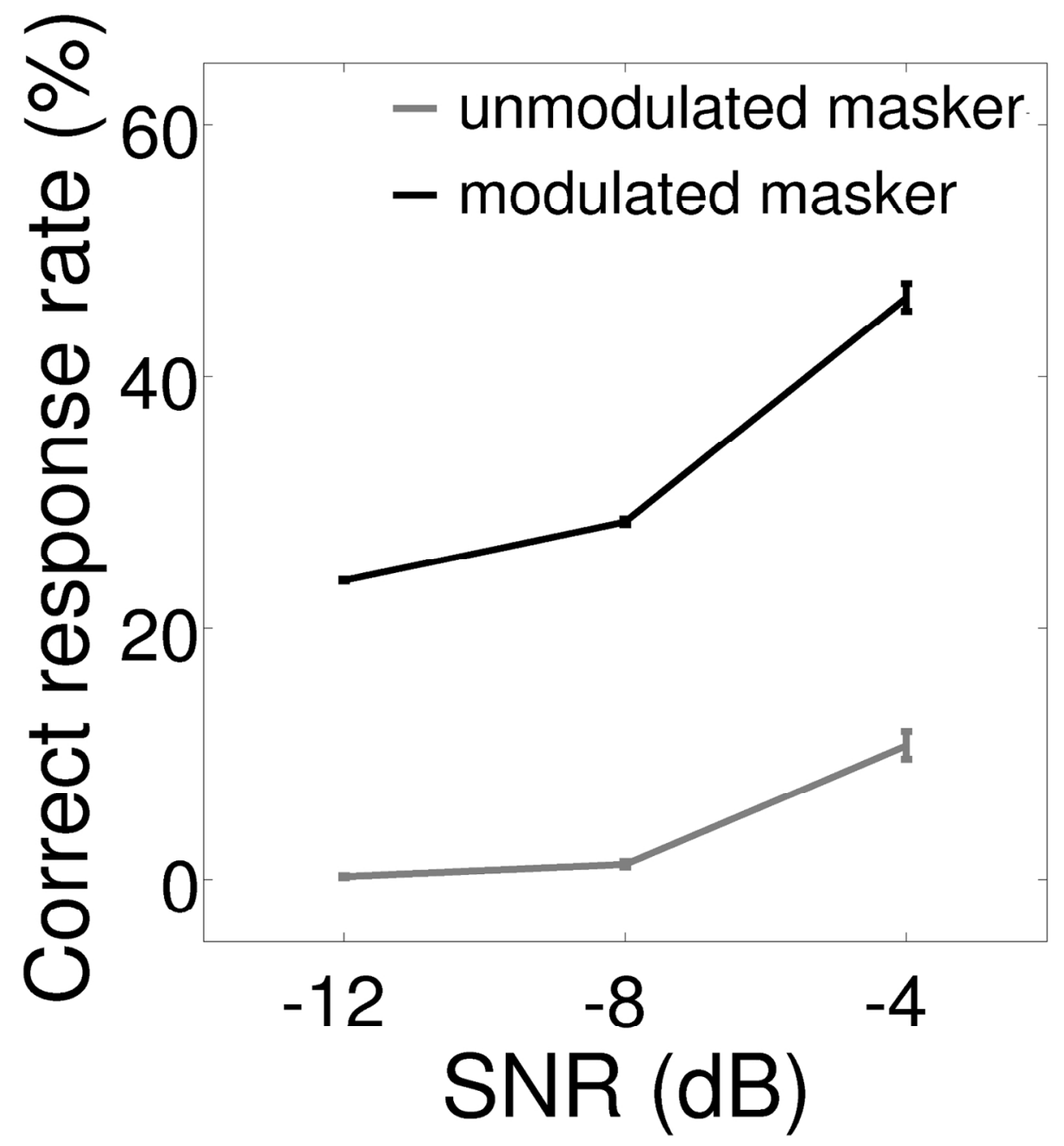

FIG. 2. Mean accuracy (\%) for keyword identification of IEEE sentences as a function of SNR. Sentences were presented in an unmodulated noise masker (grey line) and a square-wave-gated noise masker (black line). Error bars show +/- one SEM. 


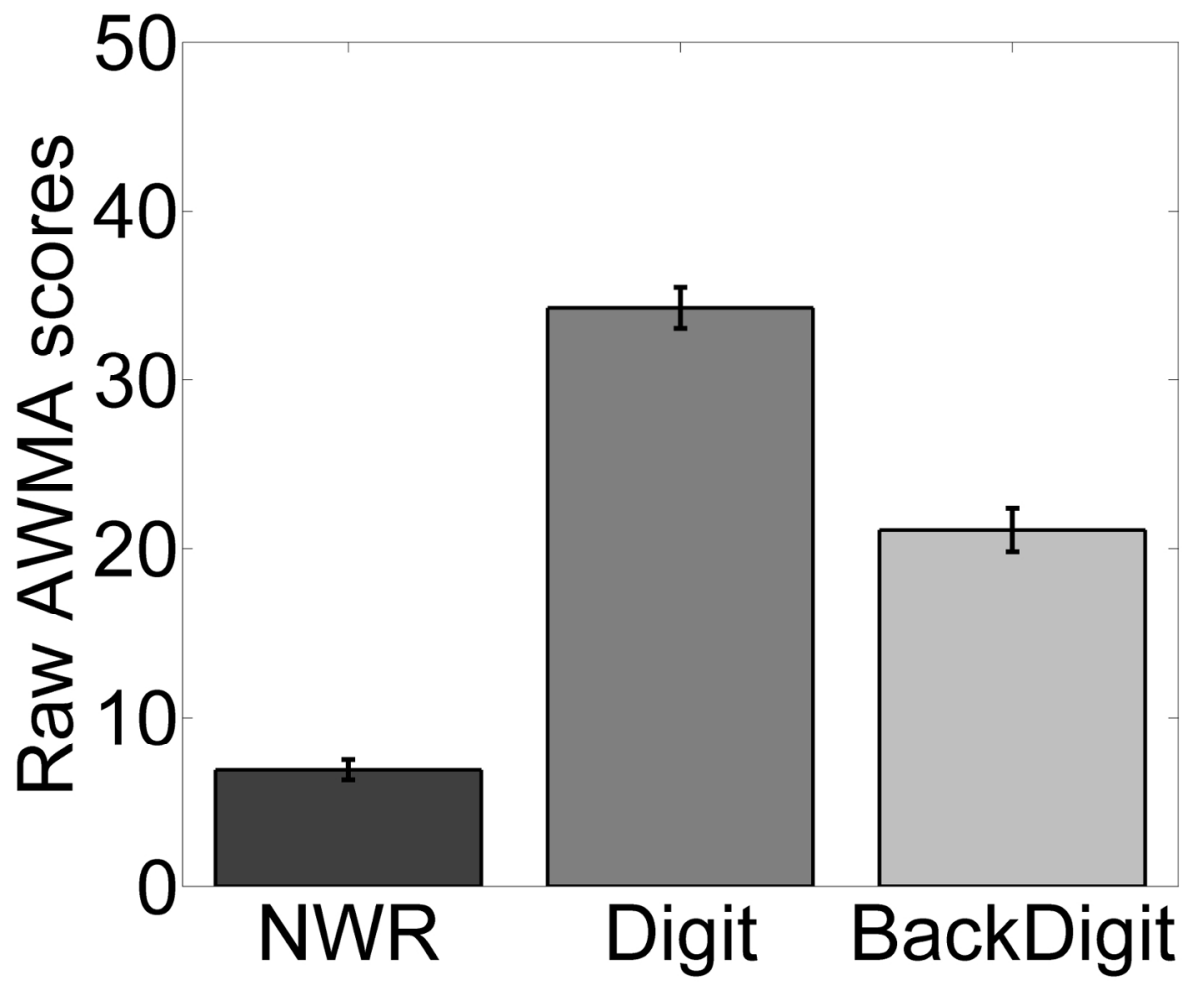

FIG. 3. Mean AWMA raw scores. Memory tests were non-word repetition (NWR), forward digit recall (Digit) and backward digit recall (BackDigit). Error bars show +/- one SEM. 


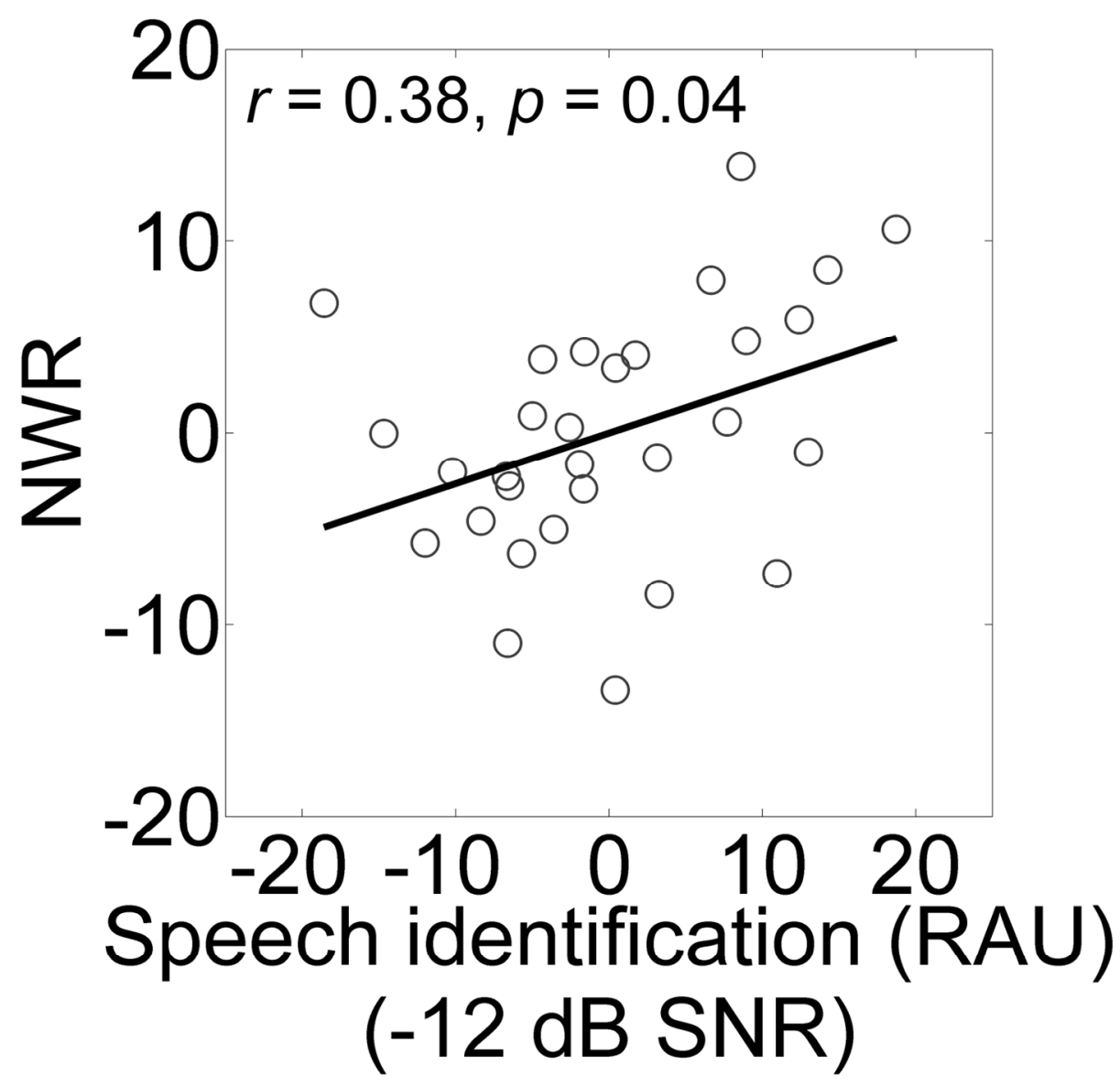

FIG. 4. Partial regression plots of the relationship between non-word repetition (NWR) and speech perception in modulated noise at $-12 \mathrm{~dB}$ SNR (Speech Identification (RAU), $-12 \mathrm{~dB}$ SNR), after controlling for the effects of Age and PTA.

$152 \times 141 \mathrm{~mm}(300 \times 300 \mathrm{DPI})$ 